\title{
Enhanced Recovery in Colorectal Surgery: Are we Going Forward or Backward?
}

ISSN: 2578-0379

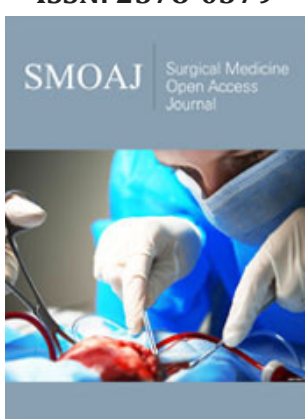

*Corresponding author: Jayesh Sagar, Department of Colorectal \& General Surgery, UK

Submission: 睯 December 20, 2019

Published: 啙January 08, 2020

Volume 3 - Issue 2

How to cite this article: Shantata J K, Jayesh S. Enhanced Recovery in Colorectal Surgery: Are we Going Forward or Backward?. Surg Med Open Acc J.3(2). SMOAJ.000559.2020. DOI: 10.31031/SMOAJ.2020.03.000559.

Copyright@ Jayesh Sagar, This article is distributed under the terms of the Creative Commons Attribution 4.0 International License, which permits unrestricted use and redistribution provided that the original author and source are credited.

\author{
Shantata J Kudchadkar and Jayesh Sagar* \\ Department of Colorectal \& General Surgery, UK
}

\begin{abstract}
Enhanced Recovery (ERAS) in Colorectal Surgery is a relatively novel concept in patient care. It involves a multidisciplinary team approach (surgeons, anesthetists, ERAS nurse, nutritionist, physiotherapist, pain team, hospital administration and patient motivation) comprising of certain key aspects in the pre, intra and post-operative settings. The whole objective of ERAS pathway is to reduce the physiological trauma to the patient and optimize organ functions, thus leading to reduced pain, post-operative complications, faster patient recovery, improvement in overall outcome, shorter hospital stays and thus, accounting for decreased health-care costs. However, there is still a need for more patient-specific, better designed large-scale, multi-centre randomized trials to study long-term impact of ERAS. A valid primary research question still remains to be answered-whether it is reasonable to apply one standard fixed protocol to everyone or modify the ERAS care to an individual patient considering various other parameters such as patient characteristics and physiology, pre-existing co-morbidities, indication \& type of surgery (emergency/elective) and compliance factors. Our aim is to highlight the importance of formulating a personalized ERAS program for certain high-risk patients, rather than adopting one rigid protocol for everyone.
\end{abstract}

Keywords: Perioperative; Laparoscopy; Prehabilitation; Colorectal

Abbreviations: ERAS: Enhanced Recovery After Surgery; LOS: Length of Stay

\section{Introduction}

Enhanced recovery After Surgery (ERAS) or "fast track surgery" protocol was first proposed by Henrik [1], Danish Professor of surgery in 1997 and was later developed further by the ERAS working group [2]. Professors Kenneth Fearon and Olle Ljungqvist added postulates to the ERAS protocol, developing the ERAS study group in 2001 and subsequent formation of the ERAS Society in 2010. The intention was to facilitate efficient and safe patient progress from admission to discharge and early rehabilitation. In 2010, ERAS Society was established, with the idea of creating international network of regional and national expert centers that encouraged ERAS protocol utilization. ERAS is based on accelerating early patient recovery following any major surgery, by decreasing the surgical trauma and the inherent body's stress response, thereby preserving individual's physiology and organ functions. It consists of 17 key components, ranging from pre-operative education, counselling and nutrition, perioperative measures including fluid optimization, active warming, oxygen administration, to postoperative early enteral nutrition, early ambulation and opioid sparing analgesia. All elements are devised based on high quality evidence $[3,4]$.

Two studies analysed the feasibility and relationship between ERAS protocol adherence and clinical outcomes in laparoscopic colorectal surgery and found that, at least 30 patients are required for a period of about 6 months, to achieve a compliance rate of $80 \%[3,4]$. They concluded that complete implementation of ERAS protocol decreases complications, re-admissions, Length of Hospital Stay (LOS) and morbidity rate. Overall, it has a positive impact on specific convalescence parameters, with significant improvement in short term outcomes. One systemic meta-analysis investigated the synergistic effect of ERAS with laparoscopy in colorectal surgery. It reported reduction in major morbidity and hospital stay in group who underwent laparoscopic procedure combined with ERAS compared to open procedure combined with ERAS. However, they concluded that the reduction was due to laparoscopic approach rather than ERAS. There was also no difference in complications between conventional care and ERAS in the laparoscopic group. These findings question the real advantage of ERAS [5]. 


\section{Personalized ERAS Program}

A recent Cochrane review analysis inferred that, though ERAS in colorectal surgery is safe with reduced overall complications and hospital stay, there was not sufficient evidence to justify its implementation as the standard of care, as major surgical complications including anastomotic leakage, paralytic ileus, intra-abdominal abscesses, adhesions and late incisional hernia were not reduced and protocol compliance in the field still remains the major problem [6]. Employing ERAS in the care of elderly can be quite challenging, due to existing comorbidities and frailty associated with age. We are all well aware of the fact, that post-operative morbidity is higher in elderly, following any major surgery. However, literature provides a substantial number of comparative studies, which support reduced morbidity with institution of ERAS in elderly [7]. But still, certain factors have to be kept in mind with regards to application of ERAS in elderly. There is inferior compliance with ERP components in elderly, as compared to younger population. There is an element of poor physiological reserve with early decompensation in elderly and early discharge from hospital can lead to extra costs for home care $[8,9]$.

Though the aims of ERAS remain the same in elderly group, end-points such as LOS, complications, re-admissions, re-operation rates and ERAS protocol adherence differ to certain extent compared to younger population. These features have to be patient specific in elderly, as they are dependent on other co-existing factors such as pre-existing anaemia, poor nutritional status, complex co-morbidities, and previous abdominal surgery [9]. These factors demand element of flexibility in the ERAS pathway. New strategies can be employed to increase the utilization of ERAS in elderly, such as preoperative geriatric specific work-up, use of pre-operative rehabilitation $[10,11]$ (comprising of nutritional assessment, protein supplementation, physiotherapy during the month preceding surgery), planning regular post-operative followup and multimodal rehabilitation program [12]. A risk assessment system should to be designed to identify high-risk patients and alter certain features of protocol, to formulate modified ERAS pathway. Thus, evolution of ERAS guidelines should be dynamic, to encourage its use in individuals with different characteristics $[13,14]$.

\section{Barriers in ERAS Implementation}

Factors such as resistance to change, non-compliance to certain aspects of ERAS, shortage of nursing staff \& health care personnel, lack of team communication \& collaboration and limited financial resources lead to hindrance in embracing ERAS into daily practice. Introducing ERAS protocol is a gradual process and must be flexible and applicable to the local situation. It is practicable only through close team co-operation \& adaptation, continuous patient \& staff education, regular evaluation of pathway with quality audits and creation of patient friendly rehabilitation environment, as proposed by Kahokehr et al. [15].

Role of ERAS in emergency settings: Increased rate of postoperative complications and prolonged hospital stays are fairly common with emergency operations as comparted to elective ones.
In emergency settings, certain ERAS items cannot be implemented, especially pre-operative components, and requires amendments based on individual case complexity [16]. ERAS utilization in emergency settings is more effective in low-risk patients [17]. A relatively relaxed ERAS program may be more suitable in emergency environment, with adoption of few changes in regular pathway. Further research is needed to fully establish the role of ERAS in decreasing major morbidity and mortality in emergency settings [18].

\section{Long-Term Outcome}

There is growing research to execute ERAS protocol in many other surgical disciplines, apart from colorectal field, including gastric, hepatic, pancreatic, esophageal, bariatric and nongastrointestinal specialties (Urology, Gynaecology, Head \& Neck Surgery and Orthopedics) [19-26]. Although recent literature has shown ERAS to be safe and practicable with satisfactory clinical outcomes and economic benefits; further research including large clinical trials is needed to study its short- and long-term effectiveness in other specialties including emergency surgery [27].

\section{Conclusion}

The aim of enhanced recovery in colorectal surgery is to alleviate the surgical stress response and reduce the end organ dysfunction, through integrated pre, intra and post-operative care. It is an important illustration of value-based care applied to surgery. A multidisciplinary team is the cornerstone for success of ERAS pathway. We emphasize that the goal of ERAS is not to discharge the patient from hospital unless patient is fit in terms of his physiology. In high-risk patients, whether the ERAS is more advantageous compared to conventional perioperative technique still remains to be answered. We suggest implementation of a relatively individualized ERAS pathway tailored to individual's characteristics.

\section{References}

1. Kehlet H (1997) Multimodal approach to control postoperative pathophysiology and rehabilitation. Br J Anaesth 78(5): 606-617.

2. Lassen K, Soop M, Nygren J, Cox PB, Hendry PO, et al. (2009) Consensus review of optimal perioperative care in colorectal surgery: Enhanced Recovery After Surgery (ERAS) group recommendations. Arch Surg 144(10): 961-969.

3. Pędziwiatr M, Kisialeuski M, Wierdak M, Stanek M, Natkaniec M, et al. (2015) Early implementation of Enhanced Recovery After Surgery (ERAS ${ }^{\circledR}$ ) protocol-compliance improves outcomes: A prospective cohort study. Int J Surg 21: 75-81.

4. Pisarska M, Pedziwiatr M, Malczak P, Major P, Ochenduszko S, et al. (2016) Do we really need the full compliance with ERAS protocol in laparoscopic colorectal surgery? A prospective cohort study. Int J Surg 36: $377-382$.

5. Spanjersberg WR, Sambeeck JDP, Bremers A, Rosman C, Laarhoven CJHM (2015) Systematic review and meta-analysis for laparoscopic versus open colon surgery with or without an ERAS programme. Surg Endosc 29(12): 3443-3453.

6. Spanjersberg WR, Reurings J, Keus F, Laarhoven CJ (2011) Fast track surgery versus conventional recovery strategies for colorectal surgery. Cochrane Database Syst Rev 2: CD007635. 
7. Launay SMV, Mathonnet M, Theissen A, Ostermann S, Raynaud SA, et al (2017) Are enhanced recovery programs in colorectal surgery feasible and useful in the elderly? A systematic review of the literature. Journal of Visceral Surgery 154(1): 29-35.

8. Baek SJ, Kim SH, Kim SY, Shin JW, Kwak JM, et al. (2013) The safety of a fast-track program after laparoscopic colorectal surgery is comparable in older patients as in younger patients. Surg Endosc 27(4): 1225-1232.

9. Yunpeng Z, Yufang X, Peng S, Daqing C, Ming X, et al. (2019) Factors associated with failure of Enhanced Recovery After Surgery (ERAS) in colorectal and gastric surgery. Scandinavian Journal of Gastroenterology 54(9): 1124-1131.

10. Cabilan CJ, Hines S, Munday J (2015) The effectiveness of rehabilitation or preoperative exercise for surgical patients: A systematic review. JBI Database Syst Rev Implement Rep 13(1): 146-187.

11. Santa MD, Clarke H, Ritvo P, Leung YW, Matthew AG (2014) Effect of totalbody rehabilitation on postoperative outcomes: A systematic review and meta-analysis. Physiotherapy 100(3): 196-207.

12. Bardram L, Funch JP, Kehlet H (2000) Rapid rehabilitation in elderly patients after laparoscopic colonic resection. Br J Surg 87(11): 15401545.

13. Compagna R, Aprea G, Rosa D (2014) Fast track for elderly patients: Is it feasible for colorectal surgery? Int J Surg 12(Suppl 2): S20-S22.

14. Scharfenberg M, Raue W, Junghans T, Schwenk W (2007) Fast-track rehabilitation after colonic surgery in elderly patients-is it feasible? Int J Colorectal Dis 22: 1469-1474.

15. Kahokehr A, Sammour T, Zargar SK, Thompson L, Hill AG (2009) Implementation of ERAS and how to overcome the barriers. Int J Surg $7(1): 16-19$.

16. Aslar AK, Ozdemir S, Mahmoudi H, Kuzu MA (2011) Analysis of 230 cases of emergent surgery for obstructing colon cancer lessons learned. J Gastrointest Surg 15(1): 110-119.

17. Lohsiriwat V (2014) Enhanced recovery after surgery vs. conventional care in emergency colorectal surgery. World J Gastroenterol 20(38): 13950-13955.
18. Paduraru M, Ponchietti L, Casas IM, Svenningsen P, Zago M (2017) Enhanced recovery after emergency surgery: A systematic review. Bull Emerg Trauma 5(2): 70-78.

19. Małczak P, Pisarska M, Piotr M, Wysocki M, Budzyński A, et al. (2017) Enhanced recovery after bariatric surgery: Systematic review and metaanalysis. Obes Surg 27: 226-235.

20. Pisarska M, Malczak P, Major P, Wysocki M, Budzynski A, et al. (2017) Enhanced recovery after surgery protocol in oesophageal cancer surgery: Systematic review and meta-analysis. PLOS ONE 12(3): e0174382.

21. Mortensen K, Nilsson M, Slim K, Schafer M, Mariette C, et al. (2014) Consensus guidelines for enhanced recovery after gastrectomy: Enhanced Recovery After Surgery (ERAS®) Society recommendations. Br J Surg 101(10): 1209-1229.

22. Chen S, Zou Z, Chen F, Huang Z, Li G (2015) A meta-analysis of fast track surgery for patients with gastric cancer undergoing gastrectomy. Ann R Coll Surg Engl 97(1): 3-10.

23. Qi S, Chen G, Cao P, Hu J, He G, et al. (2018) Safety and efficacy of Enhanced Recovery After Surgery (ERAS) programs in patients undergoing hepatectomy: A prospective randomized controlled trial. J Clin Lab Anal e22434.

24. Perinel J, Adham M (2016) ERAS and pancreatic surgery: A review. Updates Surg 68(3): 253-255.

25. Groot JJA, Ament S, Maessen J, Dejong CHC, Kleijnen JMP, et al. (2016) Enhanced recovery pathways in abdominal gynecologic surgery: A systematic review and meta-analysis. Acta Obstet Gynecol Scand 95(4): 382-395.

26. Jones EL, Wainwright TW, Foster JD, Smith JRA, Middleton RG, et al. (2014) A systematic review of patient reported outcomes and patient experience in enhanced recovery after orthopaedic surgery. Ann R Coll Surg Engl 96(2): 89-94.

27. Verheijen PM, Ven AWH, Davids PHP, Wall BJM, Pronk A (2012) Feasibility of enhanced recovery programme in various patient groups. Int J Colorectal Dis 27(4): 507-511. 